\title{
FLUID FLOW AND PARTICLE TRANSPORT THROUGH PERIODIC CAPILLARIES
}

\author{
NAZMUL ISLAM
}

(Received 20 June 2017; first published online 28 September 2017)

\begin{abstract}
2010 Mathematics subject classification: primary 76R50; secondary 76D07, 76D17, 76M15, 76R05, 76R50.

Keywords and phrases: Stokes flow, viscous vortex flow, boundary element method, forced convection, diffusion.
\end{abstract}

Fluid flow through periodic capillaries has widespread and multidisciplinary applications. In particular, recent experimental studies have recognised the potential to separate particle dispersions within microfluidic devices and nanoporous membranes. In the types of devices of interest here, a particle-laden fluid is pumped backwards and forwards through a membrane of axi-symmetric pores, whose diameter varies along their length in a periodic but longitudinally asymmetric manner. As a result, the pores have a saw-tooth profile in longitudinal cross-section. Pumping of the particulate suspension backwards and forwards results in no net flow of the fluid; however, the particles are transported along the pores from one side of the membrane to the other.

This system involves many parameters, such as the shape and geometry of the pores, the nature of the fluid (for example, viscosity), the properties of the particles (for example, their size) and the form of the applied pressure which drives the motion of the fluid. In this thesis, we aim to gain a better understanding about the underlying processes affecting particle transport and also determine which are the important parameters of the system.

A complete and definitive theoretical model for this system is intractable to solve. However, under certain assumptions, the problem can be reduced to two interconnected sub-problems. The first involves a determination of the flow field within the pores, the second concerns the motion of the particles under the influence of the underlying flow, in combination with their Brownian motion (or diffusion). As such, we first solve the appropriate hydrodynamic equations for the flow field and then use this knowledge as input for a convection-diffusion model for the evolution of the particle distribution within a pore.

Thesis submitted to the University of South Australia in November 2016; degree approved on 15 December 2016; principal supervisor Bronwyn Hajek, cosupervisors Stan Miklavcic and Lee White.

(C) 2017 Australian Mathematical Publishing Association Inc. 0004-9727/2017 \$16.00 
We draw some interesting conclusions about the hydrodynamic problem itself (see [1]). We present a systematic study investigating the effect of tube shape and geometry on the flow structure within tubes of different profiles, including both longitudinally symmetric and longitudinally asymmetric (saw-tooth) tubes. In all profiles and under certain conditions, we show that recirculation zones or eddies can form in the expansion regions of the tubes, even in the case of low Reynolds number Stokes flow considered here.

Subsequently, and armed with knowledge of the flow structure within the tubes, we develop and solve a convection-diffusion model to investigate particle transport. First, we thoroughly characterise the influence on particle transport of tube shape and geometry, particle and fluid properties, and the applied pressure profile. We find that the amplitude of the expansion region and the nature of the pressure gradient have important consequences on the amount and direction of particle transport, respectively (see [2]). Second, we qualitatively compare our results with data from experiments at both the micro- and the nanoscale. Our calculations compare favourably in some cases, particularly when both convection and diffusion are present, but some questions still remain.

The full thesis is available through the link to the research library at the University of South Australia: http://search.library.unisa.edu.au/record/UNISA_ ALMA11140946610001831.

\section{References}

[1] N. Islam, B. H. Bradshaw-Hajek, S. J. Miklavcic and L. R. White, 'The onset of recirculation flow in periodic capillaries: geometric effects', Eur. J. Mech. B/Fluids $\mathbf{5 3}$ (2015), 119-128.

[2] N. Islam, S. J. Miklavcic, B. H. Bradshaw-Hajek and L. R. White, 'Convective and diffusive effects on particle transport in asymmetric periodic capillaries', PLOS ONE (to appear), doi:10.1371/journal.pone.0183127.

NAZMUL ISLAM, Mathematics Discipline,

Science, Engineering and Technology School, Khulna University, Khulna 9208, Bangladesh

and

Phenomics and Bioinformatics Research Centre,

University of South Australia, Mawson Lakes,

South Australia, Australia

e-mail: islam_nazmul@rocketmail.com 